\title{
From meiosis to mitosis - the sperm centrosome defines the kinetics of spindle assembly after fertilization in Xenopus
}

\author{
Tommaso Cavazza ${ }^{1,2}$, Isabel Peset ${ }^{1,2, *}$ and Isabelle Vernos ${ }^{1,2,3, \ddagger}$
}

\begin{abstract}
Bipolar spindle assembly in the vertebrate oocyte relies on a selforganization chromosome-dependent pathway. Upon fertilization, the male gamete provides a centrosome, and the first and subsequent embryonic divisions occur in the presence of duplicated centrosomes that act as dominant microtubule organizing centres (MTOCs). The transition from meiosis to embryonic mitosis involves a necessary adaptation to integrate the dominant chromosome-dependent pathway with the centrosomes to form the bipolar spindle. Here, we took advantage of the Xenopus laevis egg extract system to mimic in vitro the assembly of the first embryonic spindle and investigate the respective contributions of the centrosome and the chromosomedependent pathway to the kinetics of the spindle bipolarization. We found that centrosomes control the transition from the meiotic to the mitotic spindle assembly mechanism. By defining the kinetics of spindle bipolarization, the centrosomes ensure their own positioning to each spindle pole and thereby their essential correct inheritance to the two first daughter cells of the embryo for the development of a healthy organism.
\end{abstract}

KEY WORDS: Spindle assembly, Centrosome, Xenopus, Meiosis, Embryonic mitosis

\section{INTRODUCTION}

Once every cell cycle, the bipolar spindle assembles to faithfully segregate the genetic material to the daughter cells. In most animal cells, the establishment of spindle bipolarity is aided by efficient mechanisms driving centrosome separation, thereby defining the two spindle poles and their positions. Spindle bipolarization, however, also occurs in the absence of centrosomes. A chromosome-dependent pathway triggers microtubule assembly in a centrosome-independent manner and drives microtubule organization into a bipolar spindle (Carazo-Salas et al., 1999; Heald et al., 1996). This pathway is essential in cells naturally devoid of centrosomes, like vertebrate oocytes (Dumont et al., 2007), but also in most other cell types that do contain centrosomes (Gruss et al., 2002; Kalab et al., 2006).

A crucial transition in the dynamics of spindle assembly occurs after fertilization of the vertebrate oocyte. Upon fertilization, the acentrosomal egg receives from the sperm cell the male

\footnotetext{
${ }^{1}$ Cell and Developmental Biology Programme, Centre for Genomic Regulation (CRG), Barcelona Institute of Science and Technology, Doctor Aiguader, 88 Barcelona 08003, Spain. ${ }^{2}$ Universitat Pompeu Fabra (UPF), Doctor Aiguader 88 Barcelona 08003, Spain. ${ }^{3}$ Institució Catalana de Recerca I Estudis Avançats (ICREA), Passeig de Lluis Companys 23, Barcelona 08010, Spain.

*Present address: Cancer Research UK, Manchester Institute, The University of Manchester, Wilmslow Road, Withington, Manchester M20 4BX, England, UK.

${ }^{\ddagger}$ Author for correspondence (isabelle.vernos@crg.eu)
}

D T.C., 0000-0003-2544-5340; I.V., 0000-0003-1469-9214

Received 18 November 2015; Accepted 6 May 2016 chromosomes and the single centrosome that acted as the basal body of its flagella. Therefore, the first embryonic division occurs under unique conditions that most probably require integration of the strong and dominant chromosome-dependent pathway that is supported by the egg cytoplasm with the presence of two new microtubule organizing centres (MTOCs) - the duplicated male centrosome. This integration must drive the formation of a bipolar spindle with the centrosomes correctly positioned at the spindle poles to ensure the faithful segregation of the chromosomes and the centrosomes to the first two daughter cells of the embryo. It is now clear that the correct segregation of the centrosomes is essential for the development of an adult organism because they are required for multiple functions and, in particular, for the assembly of cilia (Basto et al., 2006).

Although previous work has shown that the components and the regulation of the spindle assembly machinery transits gradually from the meiotic to the mitotic state (Courtois et al., 2012; Wilbur and Heald, 2013), it has also been proposed that a crucial transition from meiotic to early embryo spindle assembly occurs after fertilization (Kubiak et al., 2008). The dynamics of spindle assembly during this crucial transition has mostly been addressed in mice embryos. However, because in that system all the early cell divisions occur in the absence of centrosomes, it cannot provide insights into their integration in the mechanism of spindle assembly after fertilization (Courtois et al., 2012).

Here, we investigated the transition from the assembly of the meiotic acentrosomal spindle to the assembly of the embryonic spindle that occurs in the presence of centrosomes in the fertilized egg. To investigate this process further, we took advantage of the Xenopus laevis egg extract system to mimic in vitro the assembly of the first embryonic spindle and to dissect the relative contributions of the centrosomes and the chromosome-dependent pathway in the establishment of spindle bipolarity (Karsenti and Vernos, 2001).

We found that the centrosomes dictate the kinetics of spindle bipolarization over the dominant chromosome-dependent pathway. This mechanism is linked to the correct positioning of the centrosomes to the two spindle poles, thereby ensuring their faithful segregation and inheritance.

\section{RESULTS}

Centrosomes and chromosomes promote different kinetics of spindle bipolarization

Xenopus laevis females lay eggs that are naturally arrested in metaphase of meiosis II by the cytostatic factor (CSF) (Masui and Markert, 1971). Cytoplasmic extracts prepared from these eggs (CSF extracts) maintain the cell cycle arrest and provide a good experimental system for studying various aspects of spindle assembly in vitro (Karsenti and Vernos, 2001). Here, we used this system to study the transition from a chromosome-dependent spindle assembly mechanism occurring in the oocyte to a combined mechanism including the male duplicated centrosomes for the 


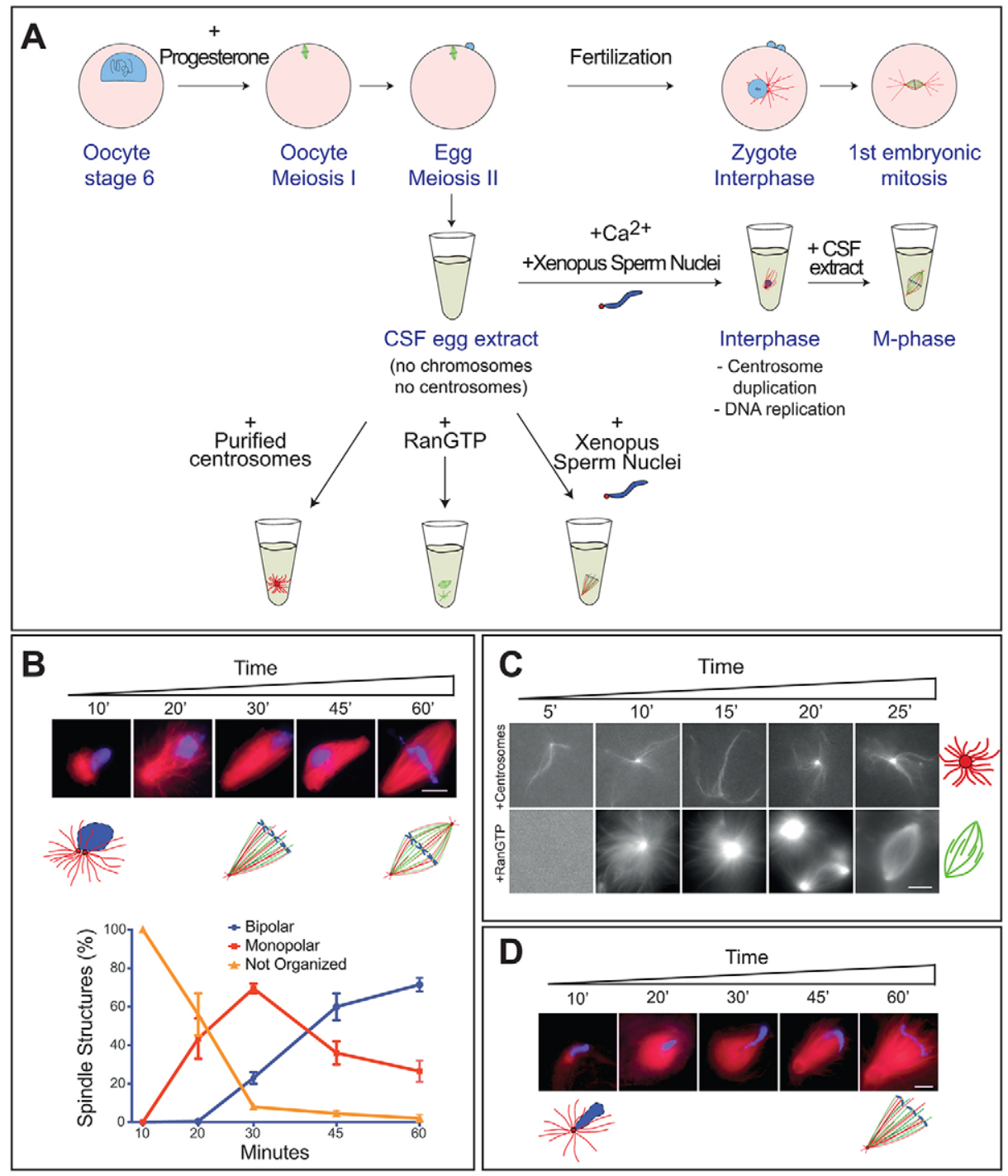

Fig. 1. The kinetics of spindle bipolarization do not correspond to the kinetics of the individual microtubule assembly pathways. (A) Schematic representation of the phases of Xenopus laevis oocyte maturation, fertilization and first embryonic division, and the corresponding egg extract manipulations for the experiments shown in B-D. The Xenopus sperm nucleus provides chromosomes (blue) and a single centrosome (red dot). (Top) Upon stimulation with progesterone, the stage-6 oocyte in the female undergoes maturation (meiosis I and II). The egg is laid, naturally arrested with CSF in metaphase of meiosis II. Because the oocyte has no centrosomes, the meiosis I and II spindles self-organize around the chromosomes. Fertilization triggers the internal release of $\mathrm{Ca}^{2+}$ in the egg that exits meiosis and enters into interphase in the presence of the male chromosomes and a single centrosome. After DNA replication and the duplication of the male centrosome, the first embryonic spindle assembles, integrating the activity of the duplicated male centrosomes and the chromosomal microtubule assembly pathway. Chromosome-dependent microtubules are in green and centrosomal microtubules are in red. The two polar bodies resulting from the asymmetric meiotic divisions are shown in blue. (Bottom) The eggs arrested in metaphase of meiosis II are collected and crushed using centrifugation to obtain the undiluted cytoplasm that does not contain chromosomes and centrosomes. This is called CSF-arrested egg extract. Addition of $\mathrm{Ca}^{2+}$ releases the CSF arrest. During interphase, the exogenously added sperm chromosomes replicate and their associated centrosome duplicates, mimicking the events occurring upon natural egg fertilization. Entry into mitosis is then triggered by addition of a small volume of CSF extract; this M-phase extract is called 'cycled extract'. The process of spindle assembly in the egg cytoplasm in the presence of the male replicated chromosomes and the associated duplicated centrosomes mimics the first embryonic spindle assembly in the embryo. The CSF extract can also be supplemented with purified components (human purified centrosomes, Xenopus sperm nuclei or Ran-GTP purified protein) to follow individually or in combination the different pathways of microtubule assembly in the M-phase egg cytoplasm. (B) Spindle assembly in cycled egg extracts goes through a monopolar intermediate. (Top) Representative images and schematic representation of the mitotic structures found at the indicated times. DNA is in blue, and tubulin is in red. Scale bar: $10 \mu \mathrm{m}$. (Bottom) Quantification of the proportion of the mitotic structures present at 10, 20, 30, 45 and 60 min after cycling into mitosis. Three categories were monitored - 'not organized' (orange), 'monopolar' (red) and 'bipolar' (blue). The proportion of monopolar spindles peaked at 30 min and later decreased. The proportion of bipolar spindles increased progressively from $30 \mathrm{~min}$ and reached a peak at 60 min. Data were obtained from two independent experiments, counting at least 100 mitotic structures per condition. Error bars are s.e.m. (C) The centrosome and the chromosome-dependent pathways follow different kinetics and assemble different type of structures. (Top) Representative images and schematic representation of microtubule asters formed by purified human centrosomes incubated in CSF extracts containing Rhodamine-tubulin. At $5 \mathrm{~min}$, the centrosome asters were already at steady state. (Bottom) Representative images and schematic representation of microtubule assemblies triggered by addition of Ran-GTP to CSF extracts containing Rhodamine-tubulin. Microtubule asters appeared at around $10 \mathrm{~min}$ and with time organize bipolar-like mini-spindles. Rhodamine-tubulin is in grey. Time in minutes is indicated on the top. Scale bar: $10 \mu \mathrm{m}$. (D) Incubation of Xenopus sperm nuclei in CSF extracts drives the formation of monopolar spindles. Representative images and schematic representation of the mitotic structures found at the indicated times. Time in minutes is indicated on the top. DNA is in blue, and tubulin is in red. Scale bar: $10 \mu \mathrm{m}$. 
assembly of the first embryonic spindle after fertilization (Fig. 1A). To mimic this transition, Xenopus sperm nuclei were added to a CSF egg extract that was released into interphase through addition of $\mathrm{Ca}^{2+}$ (Fig. 1A). Like in the fertilized egg, the sperm-associated centrosome duplicates and the DNA replicates during interphase, and upon mitotic entry after addition of CSF egg extract, the bipolar spindle assembles, recapitulating in vitro the assembly of the first embryonic spindle (hereafter referred to as 'cycled extract'; Fig. 1A). In agreement with previous reports (Boleti et al., 1996), spindle assembly in the cycled egg extract transited through a monopolar configuration that peaked after $30 \mathrm{~min}(69.5 \pm 2.5 \%$ of spindles were monopolar; mean \pm s.e.m.) and were mostly organized in a bipolar manner at $60 \mathrm{~min}(71.5 \pm 3.5 \%$ of spindles were bipolar $)$ (Fig. 1B), defining a time-dependent organization process that reaches a steady state with a given rate that we define as the kinetics of spindle bipolarization.

We first evaluated the respective contributions of the centrosomes and the chromosomes in spindle assembly by monitoring independently the kinetics of microtubule assembly and organization induced by each pathway (Fig. 1A,C). In agreement with previous reports (Verde et al., 1990), centrosomes that had been purified from human cells formed microtubule asters in CSF extracts, reaching a steady state after 5 min (Fig. 1C). However, these asters did not interact with each other, precluding the formation of more complex structures. We then supplemented a CSF extract with RanQ69L-GTP (a mutant form of the small Ran GTPase that cannot hydrolyse GTP) to mimic the chromatindependent pathway, as previously described (Carazo-Salas et al., 1999). The kinetics of microtubule assembly under these conditions were more complex than in the presence of centrosomes. The first microtubule asters appeared after $10 \mathrm{~min}$ of incubation and evolved within $5 \mathrm{~min}$ into asters that had a higher density of shorter microtubules. After $20 \mathrm{~min}$, the asters started to interact and organize bipolar structures (mini-spindles) that increased in proportion over time (Fig. 1C and data not shown) (Carazo-Salas et al., 1999; Heald et al., 1996; Sardon et al., 2008). Therefore, although both the Ran-GTP-dependent pathway and the centrosomes trigger the formation of microtubule asters, only those generated by Ran-GTP organize into bipolar structures within 20-25 min.

Altogether, these results indicate that the centrosomes and the chromatin Ran-GTP-dependent pathway individually or in combination promote different kinetics of microtubule assembly and organization. Although the centrosomes do not promote bipolarity on their own, the chromatin Ran-GTP-dependent pathway drives microtubule organization into a bipolar configuration faster than sperm nuclei, which combine both pathways (Fig. 1B,C).

To define more specifically the integration and/or contribution of the centrosome in the chromosome-driven spindle assembly pathway, which also includes the activity of the chromosome passenger complex (CPC) (Maresca et al., 2009; Sampath et al., 2004), we incubated Xenopus sperm nuclei in CSF extract (Fig. 1A,D) to follow microtubule organization in the presence of chromosomes and the single sperm centrosome. The sperm-associated centrosome is atypical in structure and protein composition in many animals and insects (Avidor-Reiss et al., 2015), although it has two centrioles and it functions as an MTOC upon fertilization. Indeed, the Xenopus sperm centrosomes nucleated microtubules efficiently soon after incubation in CSF extract, like the human centrosomes (Albee and Wiese, 2008). After $30 \mathrm{~min}$, a majority of monopolar spindles had formed around the sperm nuclei with only few bipolar spindles appearing after relatively long incubation times (Fig. 1D). Liveimaging experiments have previously reported that these bipolar spindles arise through spontaneous pole splitting or new pole selforganization (Mitchison et al., 2004). Those observations together with our results indicate that the chromosome-dependent pathway can efficiently drive bipolar spindle assembly, but the presence of a single centrosome imposes a constraint that limits this organization (Heald et al., 1997). Alternatively, or in addition, other factors - including the type of chromatin (non-replicated versus replicated) and the absence or presence of sister kinetochores - might also be at play.

Altogether, we conclude that the chromosome-dependent pathway activates one or several factors that drive the bipolar organization of microtubules, whereas the centrosomes change the kinetics of spindle bipolarization in a dominant manner. This suggests that an important adaptation occurs during the transition from meiotic to mitotic spindle assembly after fertilization, when the egg proceeds to the first embryonic division. These results also point to the importance of understanding the respective roles of the centrosomes and the chromosome-dependent pathway in spindle bipolarization in early embryos and in somatic cells.

\section{The kinetics of spindle bipolarization are adjustable}

To further explore spindle bipolarization during the first embryonic cycle, we decided to focus on the Xenopus TACC3-family member TACC3 (also known as Maskin; hereafter referred to as XTACC3). We and others have previously shown that XTACC3 promotes microtubule assembly at the centrosome in Xenopus egg extracts. Indeed, depletion of XTACC 3 results in a significant reduction of microtubules by $40 \%$ in the centrosome asters, but it does not impair bipolar spindle formation in cycled egg extracts (Kinoshita et al., 2005; Mortuza et al., 2014; Peset et al., 2005; Sardon et al., 2008). We first incubated Xenopus sperm nuclei carrying one centrosome in mock- (control) or XTACC3-depleted CSF extracts (Fig. 2A; Fig. S1A). Strikingly, after $60 \mathrm{~min}$ of incubation, a significantly higher proportion of bipolar spindles assembled in XTACC3depleted extracts than in control extracts (respectively $49.0 \pm 5.9 \%$ and $5.6 \pm 2.3 \%$; mean \pm s.e.m.; Fig. $2 \mathrm{~B}$ ). To test whether XTACC3 depletion also affects spindle bipolarization in the presence of two centrosomes, we used cycled extracts.

Interestingly, spindle bipolarization was also favoured in XTACC3-depleted cycled extracts (Fig. 2C). Indeed, $30 \mathrm{~min}$ after cycling the extract into mitosis in the presence of Xenopus sperm nuclei, a significantly higher proportion of bipolar spindles were present in XTACC3-depleted extract $(42.6 \pm 6.8 \%)$ compared to in the control extract $(19.8 \pm 5.0 \%)$. This difference was still significant after 45 min with $55.7 \pm 3.0 \%$ of bipolar spindles in XTACC3depleted extracts and $39.9 \pm 5.1 \%$ in control extracts (Fig. 2C; Fig. S1B). However, by $60 \mathrm{~min}$, the proportion of bipolar spindles in control and XTACC3-depleted extracts was similar $(64.7 \pm 7.2 \%$ and $63.0 \pm 6.1 \%$, respectively) (Fig. 2C). These phenotypes were specific for the XTACC3 depletion as adding back recombinant XTACC 3 at endogenous concentrations $(\approx 100 \mathrm{nM})$ to the depleted extracts fully rescued the kinetics of microtubule organization both in CSF and in cycled egg extracts (Figs S2A,B and S1C,D). These results suggest that the kinetics of bipolar spindle assembly can vary and that the time required for this process is an adjustable parameter. We then investigated how the depletion of XTACC 3 might facilitate bipolar spindle assembly.

Because XTACC3 has a role in microtubule stabilization (Peset et al., 2005), we first explored whether a mild destabilization of the microtubules could change the kinetics of spindle bipolarization. We therefore monitored microtubule destabilization and spindle 


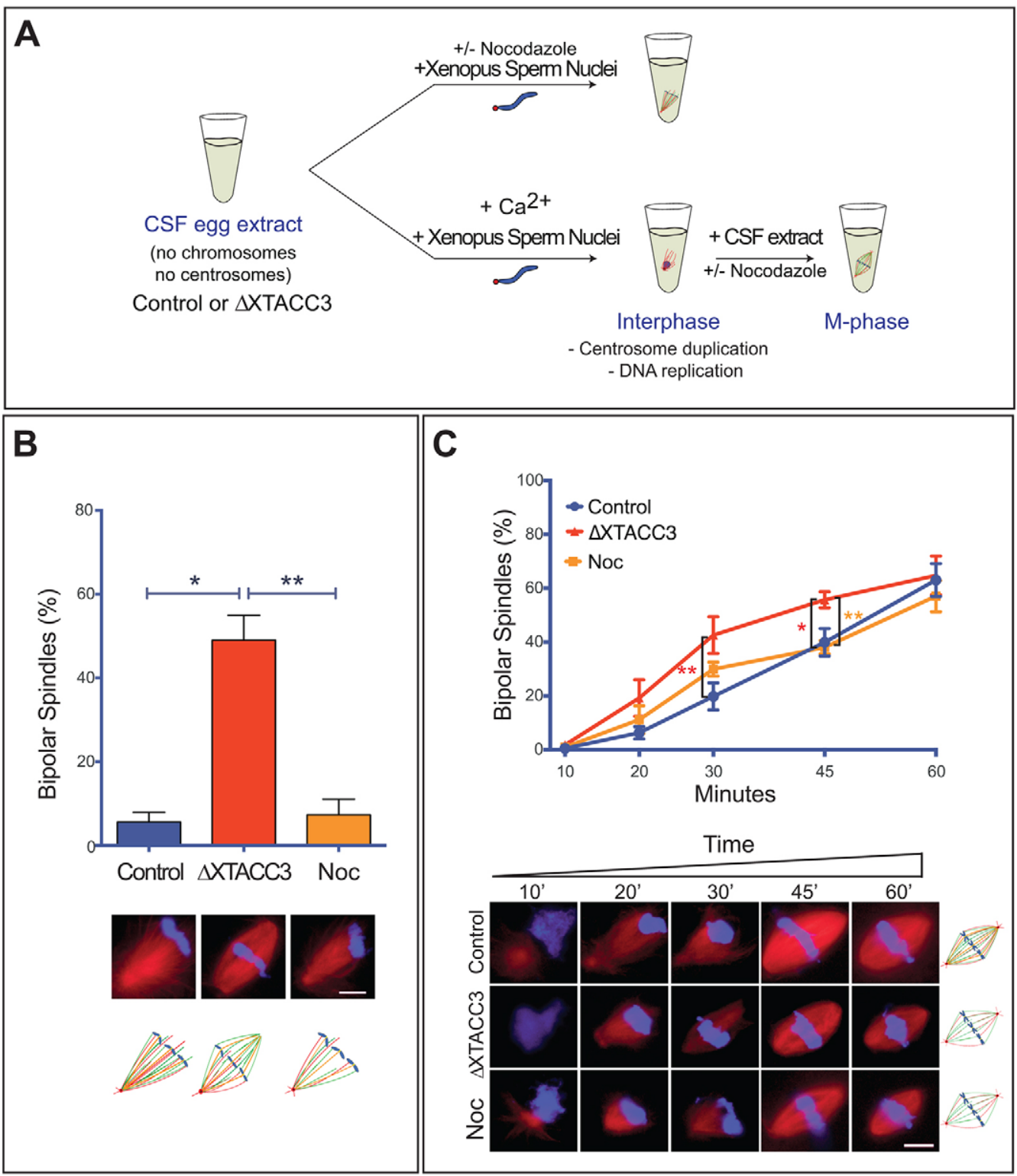

Fig. 2. Spindle bipolarization is faster in XTACC3-depleted extracts than in control extracts.

(A) Schematic representation of the experiments shown in B (upper drawing) and C (lower drawing). (B) Spindle assembly in mock-depleted (control), XTACC3-depleted ( $\triangle$ XTACC3) and nocodazolecontaining (Noc) CSF extracts. XTACC3-depleted extracts support the assembly of more bipolar spindles than control extracts \pm nocodazole. (Top) Proportion of bipolar spindles in the indicated conditions after $60 \mathrm{~min}$. Data obtained from three independent experiments, counting at least 100 mitotic structures per condition. Error bars are s.e.m. ${ }^{*} P<0.05,{ }^{*} P<0.01$ (independent two-sample $t$-test). (Bottom) Representative images of spindles assembled in the different conditions and schematic representations. DNA is in blue, and tubulin is in red. Scale bar: $10 \mu \mathrm{m}$. (C) Kinetics of spindle bipolarization in mock-depleted (control), XTACC3depleted ( $\triangle$ XTACC3) and nocodazolecontaining (Noc) cycled extracts. The bipolar spindles formed faster in the absence of XTACC3 than in control extracts \pm nocodazole. (Top) Proportion of bipolar spindles at the indicated times for each condition. Data obtained from four independent experiments, counting at least 100 mitotic structures per condition. Error bars are s.e.m. ${ }^{*} P<0.05$ indicates Control vs $\triangle$ XTACC 3 at $45 \mathrm{~min}$; ${ }^{*} P<0.01$ indicates Control vs $\triangle X T A C C 3$ at 30 min and Noc vs $\triangle \mathrm{XTACC} 3$ at $45 \mathrm{~min}$ (independent twosample $t$-test). (Bottom) Representative images of spindles, and schematic representations in the indicated conditions and at the different times. DNA is in blue, and tubulin is in red. Scale bar: $10 \mu \mathrm{m}$. assembly upon addition of different concentrations of nocodazole to the egg extract. The addition of $0.1 \mu \mathrm{M}$ nocodazole did not prevent the assembly of bipolar spindles, but the density of tubulin within them was reduced by $45.4 \pm 0.48 \%$ and their size by $31.7 \pm 0.3 \%$, indicating that microtubules were indeed partially destabilized (Fig. S3). We therefore selected this concentration for further studies. The proportion of bipolar spindles that assembled in either CSF or cycled extracts in the presence of $0.1 \mu \mathrm{M}$ nocodazole was similar to that under control conditions (Fig. 2B,C).

We conclude that the kinetics of spindle bipolarization are adjustable, but they are not directly dependent on microtubule dynamics. To investigate which parameters might define the kinetics of spindle bipolarization, we took advantage of our experimental setup to address how XTACC3 influences these kinetics.

\section{The kinetics of spindle bipolarization are not defined by K-fibre formation nor by the kinetics of the chromosome- dependent pathway}

Kinetochore fibres (K-fibres) have been shown to play a role in spindle bipolarization in somatic cells (Toso et al., 2009). Because
TACC3 has been shown to be involved in K-fibre assembly in somatic cells (Booth et al., 2011), we first examined whether $\mathrm{K}$-fibres play a role in spindle bipolarization in egg extracts. Spindle assembly was monitored in cycled extracts that had been supplemented with control IgGs (control) or antibody against Nuf2 (Fig. S1E) that have been previously shown to prevent K-fibre formation in Xenopus egg extracts (Loughlin et al., 2011) and that can be used to label the kinetochores with immunofluorescence (Fig. S4A). The quantification of the mitotic structures formed after 30,45 and $60 \mathrm{~min}$ in cycled egg extracts showed no statistical difference in the proportion of bipolar spindles formed at any time point in control and in extracts containing antibodies against Nuf2 (Fig. 3A). These results indicate that, in contrast to that in human somatic cells (Toso et al., 2009), preventing K-fibre formation in egg extracts has no influence on the spindle bipolarization kinetics. Because K-fibres account for only $5 \%$ of the total microtubules of the egg extract spindles (Ohi et al., 2007), this result is not unexpected. However, it strongly indicates that the change in bipolarization kinetics observed in XTACC3-depleted extracts is unrelated to K-fibre assembly. 
We have previously shown that XTACC3 depletion results in a delay in Ran-GTP-induced microtubule assembly in egg extracts (Sardon et al., 2008). To test whether this could explain the change of the spindle bipolarization kinetics that we observed in XTACC3depleted extracts, we monitored spindle assembly that was exclusively driven by the chromatin-dependent pathway (in the absence of centrosomes and kinetochores) by incubating chromatincoated beads (Heald et al., 1996) in mock- (control) and XTACC3depleted extracts (Figs S1F and S4B). Bipolar spindles assembled around chromatin-coated beads in control and XTACC3-depleted extracts with a similar efficiency (Fig. S4C), although consistent with previous data, the tubulin density of spindles that assembled in XTACC3-depleted extracts was significantly decreased by $25.6 \pm 2.5 \%$ (mean \pm s.e.m.) with respect to that in control spindles, without change in the spindle length (Fig. S4D). However, there was no statistical difference in the proportion of bipolar spindles present at any of the time points in control and XTACC3-depleted extracts (Fig. 3B). These results indicate that the kinetics of bipolar spindle assembly are not dictated by the kinetics of the chromosomedependent microtubule assembly pathway and strongly suggest that another mechanism governs the kinetics of spindle bipolarity establishment. This mechanism could involve the centrosomes and could be particularly relevant during the assembly of the first embryonic spindle.

\section{The centrosome controls the kinetics of spindle bipolarization}

Because XTACC3 plays a major role in promoting microtubule assembly at the mitotic centrosomes (Albee and Wiese, 2008; Kinoshita et al., 2005; O'Brien et al., 2005; Peset et al., 2005), we then focused on the putative role of the centrosome in spindle bipolarization in egg extracts.
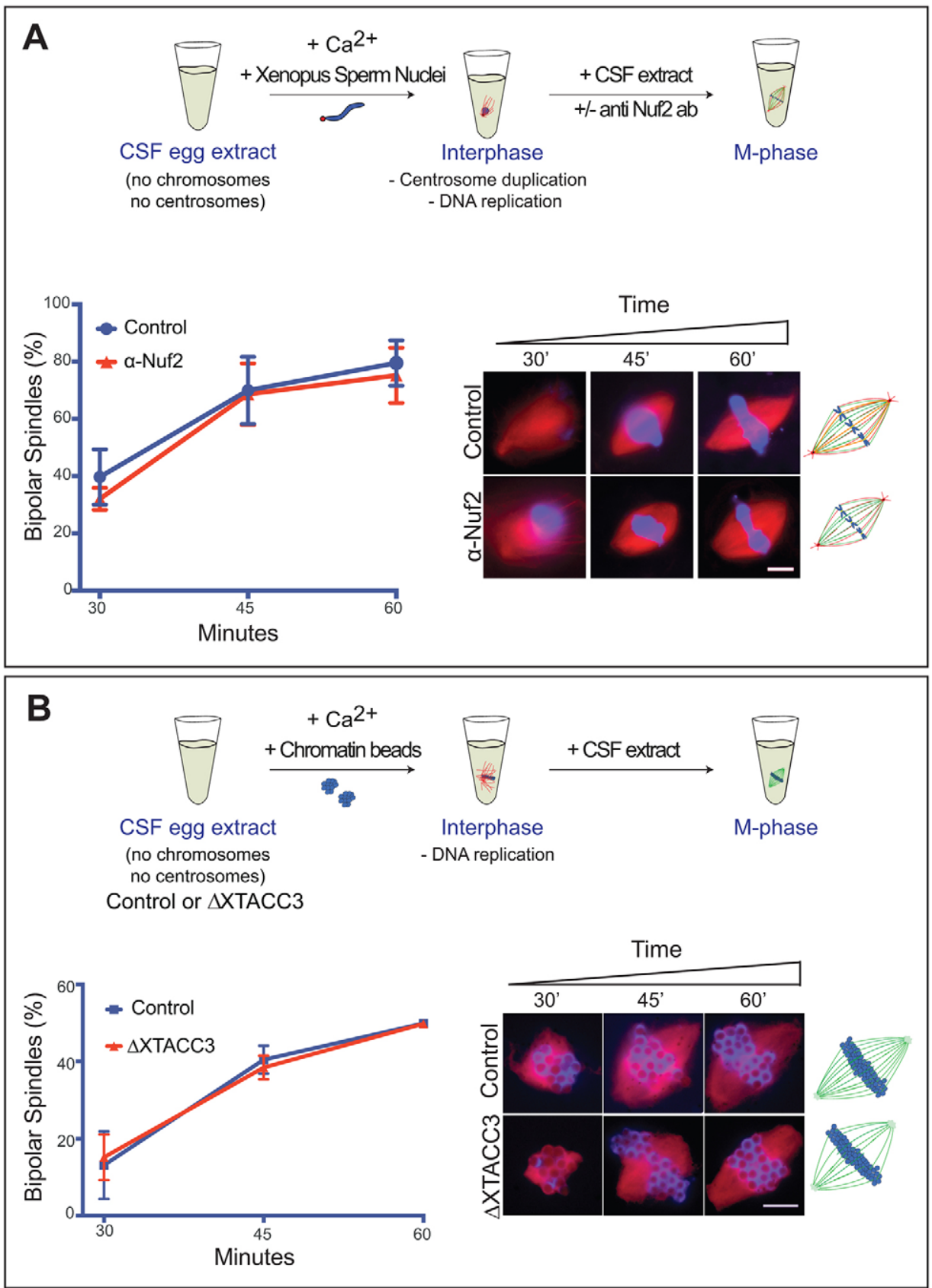

Fig. 3. The spindle bipolarization kinetics in Xenopus egg extracts do not depend on K-fibre formation or the kinetics of the chromatin-driven microtubule assembly pathway. (A) Preventing K-fibre formation by addition of anti-Nuf2 antibodies does not influence the kinetics of spindle bipolarization in cycled egg extracts. A schematic representation of the experimental setup is shown at the top. (Left) Proportion of bipolar spindles at the indicated times in control and anti-Nuf2antibody-containing extracts. Data obtained from three independent experiments, counting at least 100 mitotic structures per condition. Error bars are s.e.m. No statistically significant difference was found by independent twosample $t$-test. (Right) Representative images of the structures formed under the indicated conditions and at the indicated times ( $\mathrm{min}$ ) with the corresponding schematic representations. DNA is in blue, and tubulin is in red. Scale bar: $10 \mu \mathrm{m}$. (B) Spindles that assembled around chromatin-coated beads in mock-depleted (control) and XTACC3-depleted extracts ( $\triangle$ XTACC3) follow similar kinetics. A schematic representation of the experimental setup is shown at the top. (Left) Proportion of bipolar spindles at the indicated times in control and XTACC3-depleted extracts. Data obtained from three independent experiments, counting at least 100 mitotic structures per condition. Error bars are s.e.m. No statistically significant difference was found by independent twosample $t$-test. (Right) Representative images of the structures formed under the indicated conditions and at the indicated times ( $\mathrm{min}$ ) with the corresponding schematic representations. DNA is in blue, and tubulin is in red. Scale bar: $10 \mu \mathrm{m}$. 
We first decided to follow a complementary approach that was independent of XTACC 3 to monitor spindle bipolarization around Xenopus sperm nuclei in the absence of centrosomes. Dynein inhibition in egg extracts has been previously shown to promote the dissociation of the centrosomes from the spindle poles as well as spindle bipolarization in CSF extracts (Heald et al., 1997). We therefore inhibited Dynein by supplementing mock- (control) and XTACC3-depleted extracts with Dynamitin (also known as p50 and Dctn2) (Wittmann and Hyman, 1999).

Addition of $\mathrm{p} 50$ to CSF extracts promoted the formation of a higher proportion of bipolar spindles ( $76.3 \pm 4.2 \%$; mean \pm s.e.m.) than in control conditions. As reported above, XTACC3-depleted extracts contained more bipolar spindles $(41.4 \pm 7.7 \%)$ than control extracts $(13.6 \pm 8.6 \%)$ (Fig. 4B; Fig. S1G). Interestingly, the addition of p50 to XTACC3-depleted extracts did not significantly change the proportion of bipolar spindles $(86.1 \pm 2.0 \%)$ when compared to control extracts containing p50 (76.3 $\pm 4.2 \%)$ (Fig. 4B), suggesting no additive effects. Consistently, because $\mathrm{p} 50$ promotes the release of the centrosome from the spindle pole, the effects of p50 on spindle bipolarization were stronger than in the absence of XTACC 3 because, under these conditions, the centrosome retains some activity.

These data indicate that 'removing' the centrosome (through addition of p50) or reducing its microtubule assembly activity in CSF extracts (by depleting XTACC3) favours the self-organization of the chromosome-dependent microtubules into a bipolar configuration. These data support the idea that the male centrosome provided by the Xenopus sperm nuclei has a dominant microtubule-focusing activity (Heald et al., 1997), interfering with the bipolarization mechanism driven by the chromosome-dependent pathway in CSF extracts.

We next monitored spindle bipolarization in control and XTACC3depleted cycled egg extracts that had been supplemented with p50.

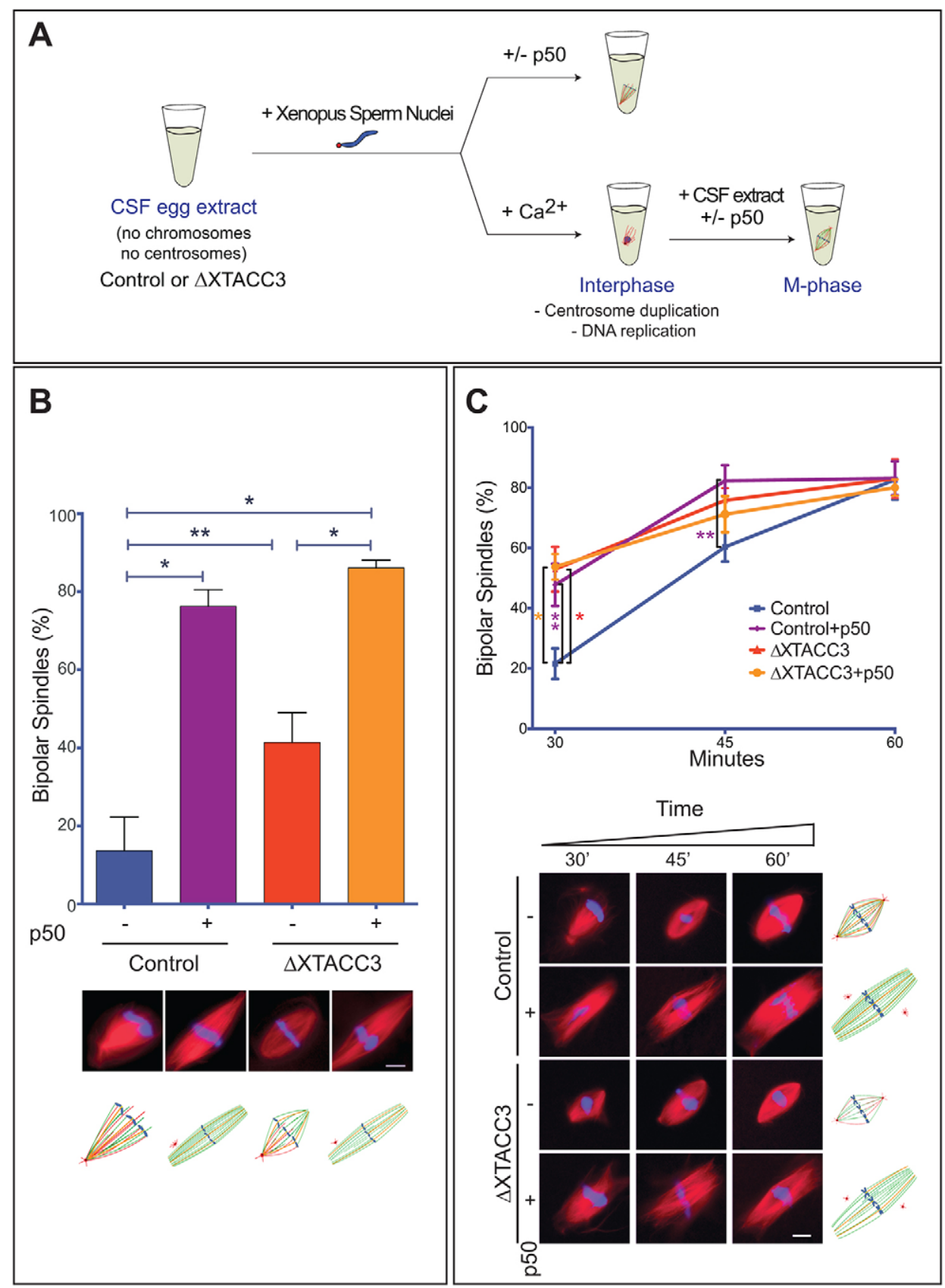

Fig. 4. Centrosome activity and number control spindle bipolarization in Xenopus egg extracts. (A) Schematic representation of the experiments shown in B (upper drawing) and $C$ (lower drawing). (B) Spindle assembly in the presence of p50 in mock-depleted (control) and XTACC3-depleted ( $\triangle$ XTACC3) CSF extracts. Addition of p50 promotes bipolar spindle assembly. (Top) The proportion of bipolar spindles after 60 min under the different conditions, as indicated. Data obtained from three independent experiments, counting at least 100 mitotic structures per condition. Error bars are s.e.m. ${ }^{*} P<0.05$, ${ }^{\star *} P<0.01$. (Bottom) Schematic representation and representative images of the spindles under the indicated conditions at the indicated times. DNA is in blue, and tubulin is in red. Scale bar: $10 \mu \mathrm{m}$. (C) Spindles assembled in cycled extracts that had been supplemented with p50 or in XTACC3-depleted extracts bipolarize faster than under mock-depleted (control) conditions. (Top) Proportion of bipolar spindles at the indicated times under the indicated conditions. Data obtained from four independent experiments, counting at least 100 mitotic structures per condition. Error bars are s.e.m. ${ }^{*} P<0.05$ is for Control vs $\triangle$ XTACC $3 \pm$ p50 at $30 \mathrm{~min}$; ${ }^{* *} P<0.01$ is for Control vs Control+p50 at $30 \mathrm{~min}$ and $45 \mathrm{~min}$ (independent two-sample $t$-test). (Bottom) Representative images and schematic representation of the spindles under the different indicated conditions at the indicated times. DNA is in blue, and tubulin is in red. Scale bar: $10 \mu \mathrm{m}$. 
Control extracts had significantly fewer bipolar spindles than p50containing extracts at $30 \mathrm{~min}$ after cycling $(21.6 \pm 5.1 \%$ in control extracts and $47.7 \pm 7.0 \%$ in extracts containing p50). This difference persisted at $45 \mathrm{~min}$ with $60.3 \pm 4.9 \%$ of bipolar spindles in control extracts and $82.3 \pm 5.2 \%$ in extracts containing p50. However, at $60 \mathrm{~min}$, the proportion of bipolar spindles was similar in control and p50-containing extracts $(82.7 \pm 6.7 \%$ and $83.1 \pm 5.6 \%$, respectively) (Fig. 4C). No significant differences were observed in control and XTACC3-depleted extracts containing p50 (Fig. 4C), suggesting no additive effects on the kinetics of bipolarization in cycled extracts.

Altogether, these data indicate that spindle bipolarization occurs more rapidly when centrosomes are removed from the spindle, suggesting that the centrosomes impose a constraint on the chromosome-dependent self-organization mechanism that drives spontaneous bipolarization. Under natural conditions, this negative constraint might be relieved upon centrosome separation.

\section{Microtubule assembly at the centrosomes is necessary for their positioning at the spindle poles}

Our data suggest that the faster kinetics of spindle bipolarization observed in XTACC3-depleted extracts are most certainly due to a relief of the negative constraint imposed by the centrosome(s). We next addressed the consequences of relieving this constraint on centrosome positioning at the spindle poles. To monitor the localization of the centrosomes in spindles that assembled in mock(control) and XTACC3-depleted cycled extracts, we performed immunofluorescence analysis with antibodies against Centrin to stain centrioles (Fig. 5A,B). We found that in control extracts, $85.4 \pm 6.5 \%$ (mean \pm s.e.m.) of the spindles had one centrosome associated to each spindle pole. In contrast, $49.7 \pm 6.6 \%$ of the bipolar spindles that had assembled in XTACC3-depleted extracts had centrosome-positioning defects. These defects included the presence of only one centrosome at one spindle pole $(29.0 \pm 2.3 \%)$, two centrosomes at one pole $(9.9 \pm 2.1 \%)$ or no associated centrosome at all (10.9 $\pm 2.4 \%)$ (Fig. 5B).

These data indicate that centrosomes generating few microtubules get either randomly associated to a spindle pole or are lost. This suggests that spindle bipolarization and centrosome positioning at the spindle poles are two closely intertwined processes - by imposing the kinetics of bipolar organization concomitantly with their dominant microtubule-focusing activity, the centrosomes ensure their correct positioning to each spindle pole. This
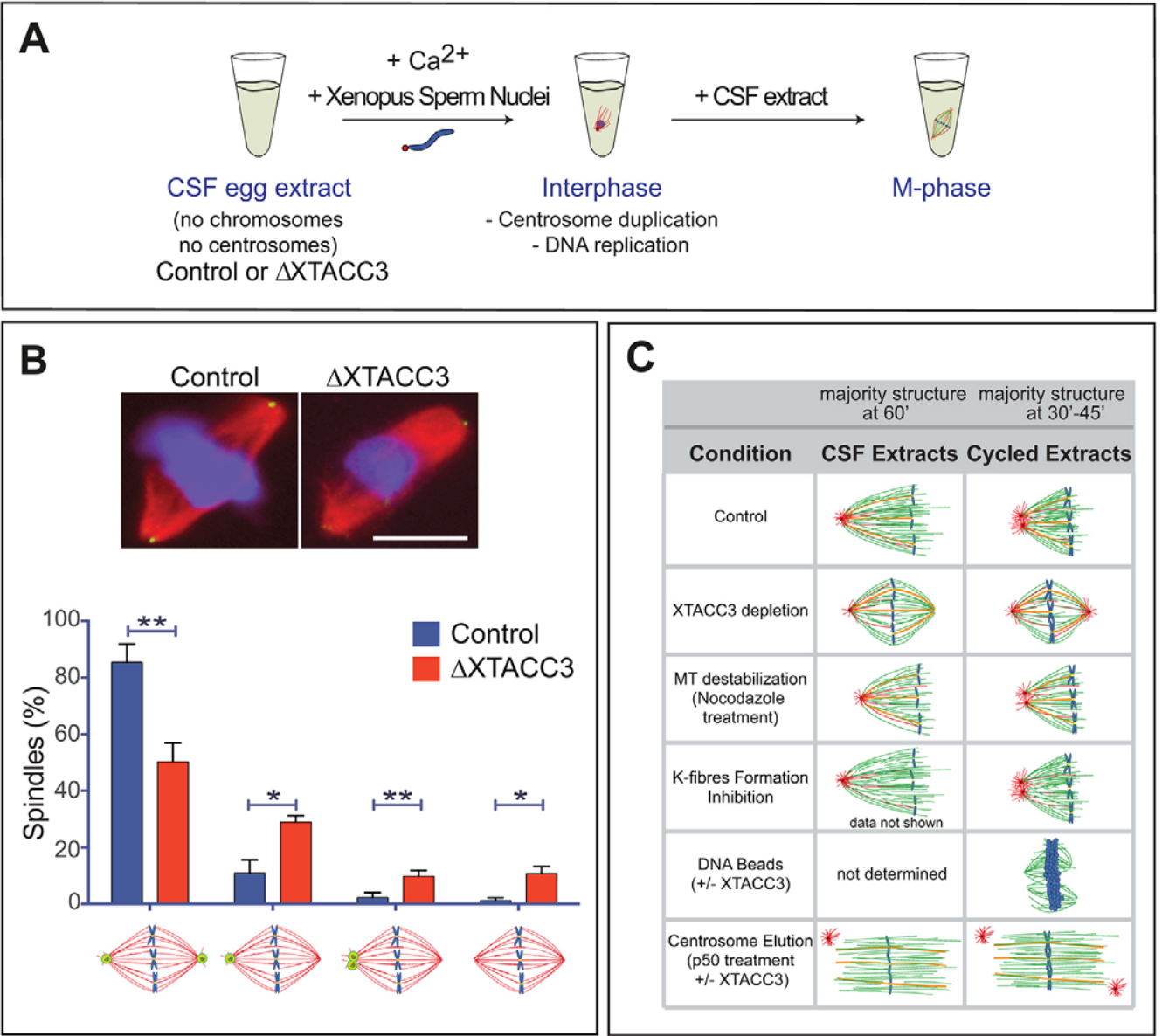

Fig. 5. A minimal microtubule assembly activity of the centrosomes is required for their correct positioning at the two spindle poles. (A) Schematic representation of the experimental setup. (B) Top: representative immunofluorescence images of spindles that assembled in mock-depleted (control) and XTACC3-depleted extracts ( $\triangle$ XTACC3). DNA is in blue, Centrin in green and tubulin in red. Scale bar: $10 \mu \mathrm{m}$. Bottom: graph showing the proportion of spindles assembled in mock-depleted (control, blue) and XTACC3-depleted extracts ( $\triangle$ XTACC3, red). The drawings below the graph show the different categories that were quantified - spindles with one centrosome at each spindle pole, spindles with one centrosome at only one of the two spindle poles, spindles with two centrosomes at only one of the two spindle poles and spindles with no associated centrosome. Data obtained from three independent experiments, counting at least 100 mitotic structures per condition. Error bars are s.e.m. ${ }^{*} P<0.05$, ${ }^{* *} P<0.01$ (independent two-sample $t$-test). (C) Table summarizing the results presented in this study. The drawings show the most abundant structure formed after 60 min of incubation in CSF extract following $30-45$ min of incubation in cycled extracts. 
mechanism thereby ensures the correct inheritance of the centrosomes in the first daughter cells and therefore the development of the fertilized egg into a healthy embryo.

\section{DISCUSSION}

We present here a series of experiments performed in Xenopus egg extracts to address the transition from an acentrosomal spindle assembly mechanism driven by the chromosome-dependent pathway to a combined mechanism that includes the centrosome provided by the male gamete upon fertilization (Fig. 1A).

Our data suggest that, in Xenopus laevis, the combination of the centrosome and the chromosome-dependent pathways that occurs upon fertilization impacts the kinetics of spindle bipolarization (Fig. 5C). By specifically targeting the centrosomes using two independent and complementary approaches (XTACC3 depletion and p50 addition), we provide data that strongly suggest that centrosomes have a clear role in the transition from meiotic to mitotic spindle assembly. This transition is not gradual for aspects of the mechanism concerning the bipolarization of the spindle, which is in contrast with what has been shown in mice (Courtois et al., 2012). In fact, we found that, as in somatic cells (Tanenbaum and Medema, 2010), the activity of the centrosomes in promoting microtubule assembly is crucial for establishing the kinetics of spindle bipolarization during this transition (Fig. 5C), ensuring their concomitant and correct association with the two spindle poles (Fig. 5B). In contrast, preventing K-fibre formation or altering the dynamics of the chromosome-dependent pathway in this early embryonic system does not influence the spindle bipolarization kinetics (Fig. 5C). This is somehow surprising because the chromosome-dependent pathway has a dominant role in microtubule assembly in the Xenopus egg extract system (Heald et al., 1996) and its activity significantly alters the kinetics of spindle bipolarization in oocytes (Dumont et al., 2007).

Our results indicate that the centrosomes need to retain a minimal microtubule assembly activity in order to block the spontaneous spindle bipolarization promoted by the chromosome-dependent pathway. This might provide the time for centrosome separation to occur, ensuring that the centrosomes get correctly positioned at each spindle pole, thereby ensuring their faithful segregation during the first embryonic cell division.

These data fit nicely also with the observations that, in the early divisions of the Xenopus embryos, the centrosomes separate at the end of the previous cell division, favouring fast bipolar spindle assembly (Wuhr et al., 2010). Thus, the early separation of the centrosomes might be necessary to avoid the transition through a monopolar spindle and to ensure both fast bipolar spindle assembly and the correct association of the centrosomes to the spindle poles. The early centrosome separation and the fast spindle assembly would be particularly important in the Xenopus early embryos, where the spindle assembly checkpoint is inactive and the cell cycle clock is determined by the oscillation of the maturation-promoting factor (MPF) activity (Newport and Kirschner, 1984).

In this respect, the Xenopus early embryonic cell cycle is different from that of mammals, which have an active spindle assembly checkpoint that is, at least, partially active (Kubiak et al., 2008). Most of the information concerning the dynamics of spindle assembly in early embryos has been obtained using mice as model system (Courtois et al., 2012). However, the early stages of development of rodents is peculiar because the fertilized egg does not contain any centrosome that is generated de novo after the 16-cell stage (Courtois et al., 2012). It will be important to test whether, in embryos of mammals that contain centrosomes, the kinetics of spindle assembly are determined by the centrosomes and whether this is relevant for the inheritance of centrosomes in all tissues.

The centrosome is a very versatile organelle that supports many cellular functions. Although centrosomes are in fact dispensable for spindle assembly, they are important for other aspects of cell division, such as defining spindle orientation and asymmetric cell divisions. Our data indicate that, when present, the centrosomes define the timing of spindle bipolarization, one of the crucial parameters that influence the duration of mitosis.

The centrosomes are also necessary in the developing embryo and the adult organism because they carry the centrioles that are required for cilia assembly. Although flies can develop without centrosomes (Basto et al., 2006), the adult males are sterile, and both males and females have severe problems owing to the lack of cilia in type-I mechanosensory neurons, the only ciliated Drosophila cell type (Basto et al., 2006). In mammals, centrioles are necessary in almost all cell types, and the correct inheritance of centrosomes is therefore essential for healthy development.

Altogether, our results indicate that the male centrosome is crucial in order to favour the transition from meiotic to mitotic spindle assembly, ensuring the correct development of a healthy organism.

\section{MATERIALS AND METHODS Xenopus laevis egg extracts, spindle assembly and aster formation}

Xenopus laevis female and male frogs were purchased from Nasco and were used at an age between 1 and 3 years. All experiments involving animals were performed according to standard protocols approved by the ethical committee of the Parc de Recerca Biomédica of Barcelona, Barcelona, Spain. CSF egg extracts were prepared as described previously (Murray, 1991). To visualize microtubules, Rhodamine-labelled tubulin was added to the egg extract to a final concentration of $\approx 0.2 \mathrm{mg} / \mathrm{ml}$. Spindle assembly in CSF extract was performed by incubating Xenopus sperm nuclei $(500$ nuclei/ $\mu \mathrm{l})$ in CSF extract for $60 \mathrm{~min}$ at $20^{\circ} \mathrm{C}$. For cycled spindle assembly, CSF extract supplemented with Xenopus sperm nuclei was released into interphase by addition of $0.4 \mathrm{mM} \mathrm{Ca}^{2+}$. After $90 \mathrm{~min}$ of incubation at $20^{\circ} \mathrm{C}$, the interphase extract was cycled back into mitosis by adding one volume of CSF extract. After $60 \mathrm{~min}$ (or when indicated), spindle assembly was monitored by 'squashing' $1 \mu \mathrm{l}$ of the reaction and $3 \mu \mathrm{l}$ of fix solution [11\% formaldehyde, $48 \%$ glycerol, $1 \mu \mathrm{g} / \mathrm{ml}$ Hoechst 33342 in CSF-XB buffer (10 mM Hepes, $100 \mathrm{mM} \mathrm{KCl,} 0.1 \mathrm{mM} \mathrm{CaCl}$, $2 \mathrm{mM} \mathrm{MgCl}_{2}, 50 \mathrm{mM}$ sucrose, $5 \mathrm{mM}$ EGTA)] between a $18 \times 18 \mathrm{~mm}$ coverslip and a glass slide. For immunofluorescence analysis, the spindle reactions were resuspended in spindle dilution buffer (30\% glycerol, $1 \%$ Triton-X100, BRB80) and spun down (20 min, $\left.3200 \mathrm{~g}, 20^{\circ} \mathrm{C}\right)$ on a coverslip through a 4-ml spindle cushion (40\% glycerol, BRB80). Coverslips were recovered, washed in PBS and fixed for $10 \mathrm{~min}$ in freezing-cold methanol.

Chromatin-coated beads were prepared by cutting the MCP1 plasmid with HindIII. The purified DNA was biotinylated by extending the overhangs using the Klenow exo-fragment in the presence of biotinylated dATP and normal dCTP, dGTP and dUTP. The biotinylated DNA was added to washed Streptavidin Dynabeads M280 (Invitrogen) in the proportion of $2 \mu \mathrm{l}$ of beads per $\mu \mathrm{g}$ of DNA in the presence of coupling buffer (PVA 2.5\%, NaCl 1 M, EDTA 2 mM, Tris-HCl 50 mM, pH 8). After overnight incubation on a rotating wheel at room temperature, beads were retrieved. DNA loading was estimated by measuring the unbound DNA at $260 \mathrm{~nm}$. Beads were resuspended in Bead Buffer (2 M NaCl, 1 mM EDTA, $10 \mathrm{mM}$ Tris- $\mathrm{HCl}, \mathrm{pH} 7.6)$ to give a final concentration of $0.2 \mu \mathrm{g}$ DNA per $\mu \mathrm{l}$ of preparation. DNA-coated beads were chromatinized (histone assembly and/or modification) through incubation at $20^{\circ} \mathrm{C}$ in ten volumes of CSF extract for $4 \mathrm{~h}$. 30- $\mu \mathrm{l}$ aliquots were fast-frozen in liquid nitrogen. To promote assembly of spindles, aliquots were rapidly thawed and the 
supernatants discarded, and the chromatin beads were resuspended in $20-50 \mu \mathrm{l}$ of fresh CSF extract. After $10 \mathrm{~min}$ at $20^{\circ} \mathrm{C}$, the extract was cycled into interphase and processed as described for cycled spindle assembly around Xenopus sperm nuclei.

Centrosomes purified from human KE37 cells, as previously described (Bornens and Moudjou, 1998), were incubated in CSF extracts at $20^{\circ} \mathrm{C}$, and the resulting microtubule asters analyzed by squashing under a coverslip. KE-37 cells (human, leukaemia, acute lymphoblastic T cell) were originally obtained from Eric Karsenti's laboratory (EMBL, Heidelberg, Germany) and were routinely tested for contamination. Recombinant RanQ69L-GTP was added to CSF extracts at the saturating concentration of $15 \mu \mathrm{M}$ (Caudron et al., 2005). The reactions were incubated at $20^{\circ} \mathrm{C}$ for the indicated time and analysed by squashing.

XTACC 3 depletion experiments were performed using three successive rounds of XTACC3 immunoprecipitation, as described previously (Peset et al., 2005).

Spindle assembly in the presence of nocodazole (Sigma) was performed by adding the drug to a final concentration of $0.1 \mu \mathrm{M}$. Spindle assembly in the presence of anti-Nuf2 antibody was performed by addition of the antibody at the maximum possible concentration (1:10 dilution), as previously described (Loughlin et al., 2011). Rabbit generic IgGs were added to similar levels, calibrating the loading by performing western blot analyses. p50 was added to the extract at $\approx 1 \mu \mathrm{g} / \mu \mathrm{l}$ (Wittmann and Hyman, 1999).

\section{Immunofluorescence}

Blocking and antibody dilution buffer was $2 \%$ BSA (Sigma) with $0.1 \%$ Triton-X100 (Sigma). Coverslips were mounted in 10\% Mowiol (Calbiochem) in $0.1 \mathrm{M}$ Tris- $\mathrm{HCl}$ at $\mathrm{pH} 8.2$ with $25 \%$ glycerol (Merck) Samples were visualized with a $40 \times$ objective on an inverted DMI- 6000 Leica wide-field fluorescent microscope. For Centrin staining, cycled extracts containing spindles (after $60 \mathrm{~min}$ of incubation at $20^{\circ} \mathrm{C}$ ) were supplemented with $0.5 \mu \mathrm{M}$ nocodazole and 15 min later spun down onto coverslips. The samples were fixed in methanol at $-20^{\circ} \mathrm{C}$ for no longer than $5 \mathrm{~min}$. The blocking and antibody incubation buffer contained 5\% BSA. Samples were visualized with a $100 \times$ objective on an inverted DMI-6000 Leica wide-field fluorescent microscope. Pictures were acquired with the Leica Application Suite software. Images were processed and mounted using Adobe Photoshop CS5.1 (Adobe).

\section{Western blot}

Blots were developed using Alexa-Fluor-680- (Invitrogen) and IRdye$800 \mathrm{CW}$ - (LI-COR) labelled antibodies and analysed using the Odyssey Infrared imaging system.

\section{Antibodies}

Polyclonal rabbit antibodies anti-XTACC 3 and anti-GST were produced inhouse against recombinant proteins His-XTACC 3 and GST, respectively (Peset et al., 2005). The affinity-purified antibodies were used at $10 \mu \mathrm{g} / \mathrm{ml}$ for immunofluorescence analyses, and at $1 \mu \mathrm{g} / \mathrm{ml}$ for western blotting. The anti-Nuf2 antibody was a gift from Todd Stukenberg (University of Virginia, VA) (Loughlin et al., 2011) and was added to the extracts at 1:10 dilution. For immunofluorescence, it was used at 1:800. The 135 and 137 mouse monoclonal anti-XTACC3 antibodies are custom-made antibodies generated by injecting mice with recombinant His-XTACC3 and were used at 1:20 for immunofluorescences and at 1:200 for western blots (Mortuza et al., 2014). Generic rabbit IgGs were purchased from Sigma (I5006-50MG). The monoclonal anti-Centrin antibody was purchased from Merck Chemicals and Life Science (clone 20H5) and used for immunofluorescence at 1:1000.

Secondary anti-rabbit and anti-mouse antibodies conjugated to AlexaFluor- $488,-568$ or -680 (Invitrogen), or to IRdye $800 \mathrm{CW}$ (LI-COR) were used at 1:1000 for immunofluorescence and 1:10,000 for western blot.

\section{Recombinant proteins}

RanQ69L was purified and loaded with GTP as previously described (Brunet et al., 2004). p50 was purified as described previously (Wittmann and Hyman, 1999). GST-XTACC3 and His-TACC3 was purified as described previously (Peset et al., 2005).

\section{Quantifications and statistics}

The sample size quantified in each experiment and the number of experimental replicates are reported in the figure legends. For the quantifications of the spindle structures in CSF extracts and the centrosome distribution in mock- and XTACC3-depleted extracts, the investigator was blinded to the experiments. Mitotic structures forming around more than one Xenopus sperm nucleus were not considered for quantification. For the measurement of spindle length and microtubule density in spindles assembled in egg extracts, the pictures were taken using the $40 \times$ objective and identical camera settings. Images were opened using ImageJ, and spindle areas were drawn manually using the freehand selection tool. Spindle length was measured using the straight-line tool. The statistical analysis of the data was done by performing an independent two-sample $t$-test in Prism 6 (Graphpad) or Microsoft Excel (Microsoft).

\section{Acknowledgements}

We are thankful to Nuria Mallol and Jacobo Cela for their excellent technical help with protein purification, antibody purification and frog handling. We thank Todd Stukenberg (University of Virginia, USA) for the gift of the anti-Nuf2 antibody. We are grateful to the Vernos lab members for discussions during the development of the project.

\section{Competing interests}

The authors declare no competing or financial interests.

\section{Author contributions}

T.C. designed, carried out and analyzed all experiments; I.P. did the initial experiments that motivated this project. T.C., I.P. and I.V. conceived the project and prepared the manuscript; I.V. supervised the study.

\section{Funding}

T.C. was supported by a Formación de Personal Investigador (FPI) fellowship (Ministerio de Economía y Competitividad) [grant number BES-2010-031355]. This work was supported by the Ministerio de Economía y Competitividad [grant numbers BFU2009-10202 and BFU2012-37163]. We acknowledge the support of the Spanish Ministerio de Economía y Competitividad programme 'Centro de Excelencia Severo Ochoa 2013-2017' [grant number SEV-2012-0208].

\section{Supplementary information}

Supplementary information available online at

http://jcs.biologists.org/lookup/doi/10.1242/jcs.183624.supplemental

\section{References}

Albee, A. J. and Wiese, C. (2008). Xenopus TACC3/maskin is not required for microtubule stability but is required for anchoring microtubules at the centrosome. Mol. Biol. Cell 19, 3347-3356.

Avidor-Reiss, T., Khire, A., Fishman, E. L. and Jo, K. H. (2015). Atypical centrioles during sexual reproduction. Front. Cell Dev. Biol. 3, 21.

Basto, R., Lau, J., Vinogradova, T., Gardiol, A., Woods, C. G., Khodjakov, A. and Raff, J. W. (2006). Flies without centrioles. Cell 125, 1375-1386.

Boleti, H., Karsenti, E. and Vernos, I. (1996). Xklp2, a novel Xenopus centrosomal kinesin-like protein required for centrosome separation during mitosis. Cell $\mathbf{8 4}$ 49-59.

Booth, D. G., Hood, F. E., Prior, I. A. and Royle, S. J. (2011). A TACC3/ch-TOG clathrin complex stabilises kinetochore fibres by inter-microtubule bridging EMBO J. 30, 906-919.

Bornens, M. and Moudjou, M. (1998). Studying the composition and function of centrosomes in vertebrates. Methods Cell Biol. 61, 13-34

Brunet, S., Sardon, T., Zimmerman, T., Wittmann, T., Pepperkok, R., Karsenti, E. and Vernos, I. (2004). Characterization of the TPX2 domains involved in microtubule nucleation and spindle assembly in Xenopus egg extracts. Mol. Biol. Cell 15, 5318-5328.

Carazo-Salas, R. E., Guarguaglini, G., Gruss, O. J., Segref, A., Karsenti, E. and Mattaj, I. W. (1999). Generation of GTP-bound Ran by RCC1 is required for chromatin-induced mitotic spindle formation. Nature 400, 178-181.

Caudron, M., Bunt, G., Bastiaens, P. and Karsenti, E. (2005). Spatial coordination of spindle assembly by chromosome-mediated signaling gradients. Science $\mathbf{3 0 9}$ 1373-1376.

Courtois, A., Schuh, M., Ellenberg, J. and Hiiragi, T. (2012). The transition from meiotic to mitotic spindle assembly is gradual during early mammalian development. J. Cell Biol. 198, 357-370.

Dumont, J., Petri, S., Pellegrin, F., Terret, M.-E., Bohnsack, M. T., Rassinier, P., Georget, V., Kalab, P., Gruss, O. J. and Verlhac, M.-H. (2007). A centriole- and 
RanGTP-independent spindle assembly pathway in meiosis I of vertebrate oocytes. J. Cell Biol. 176, 295-305.

Gruss, O. J., Wittmann, M., Yokoyama, H., Pepperkok, R., Kufer, T. and Silljé, H (2002). Chromosome-induced microtubule assembly mediated by TPX2 is required for spindle formation in HeLa cells. Nat. Cell Biol. 4, 871-879.

Heald, R., Tournebize, R., Blank, T., Sandaltzopoulos, R., Becker, P., Hyman, A and Karsenti, E. (1996). Self-organization of microtubules into bipolar spindles around artificial chromosomes in Xenopus egg extracts. Nature 382, 420-425.

Heald, R., Tournebize, R., Habermann, A., Karsenti, E. and Hyman, A. (1997) Spindle assembly in Xenopus egg extracts: respective roles of centrosomes and microtubule self-organization. J. Cell Biol. 138, 615-628.

Kalab, P., Pralle, A., Isacoff, E. Y., Heald, R. and Weis, K. (2006). Analysis of a RanGTP-regulated gradient in mitotic somatic cells. Nature 440, 697-701.

Karsenti, E. and Vernos, I. (2001). The mitotic spindle: a self-made machine. Science 294, 543-547.

Kinoshita, K., Noetzel, T. L., Pelletier, L., Mechtler, K., Drechsel, D. N. Schwager, A., Lee, M., Raff, J. W. and Hyman, A. A. (2005). Aurora A phosphorylation of TACC3/maskin is required for centrosome-dependent microtubule assembly in mitosis. J. Cell Biol. 170, 1047-1055.

Kubiak, J. Z., Ciemerych, M. A., Hupalowska, A., Sikora-Polaczek, M. and Polanski, Z. (2008). On the transition from the meiotic to mitotic cell cycle during early mouse development. Int. J. Dev. Biol. 52, 201-217.

Loughlin, R., Wilbur, J. D., McNally, F. J., Nedelec, F. J. and Heald, R. (2011) Katanin contributes to interspecies spindle length scaling in Xenopus. Cell 147 1397-1407.

Maresca, T. J., Groen, A. C., Gatlin, J. C., Ohi, R., Mitchison, T. J. and Salmon, E. D. (2009). Spindle assembly in the absence of a RanGTP gradient requires localized CPC activity. Curr. Biol. 19, 1210-1215.

Masui, Y. and Markert, C. L. (1971). Cytoplasmic control of nuclear behavior during meiotic maturation of frog oocytes. J. Exp. Zool. 177, 129-145.

Mitchison, T. J., Maddox, P., Groen, A., Cameron, L., Perlman, Z., Ohi, R., Desai, A., Salmon, E. D. and Kapoor, T. M. (2004). Bipolarization and poleward flux correlate during Xenopus extract spindle assembly. Mol. Biol. Cell 15, 5603-5615.

Mortuza, G. B., Cavazza, T., Garcia-Mayoral, M. F., Hermida, D., Peset, I., Pedrero, J. G., Merino, N., Blanco, F. J. and Lyngsø, J. (2014). XTACC3-
XMAP215 association reveals an asymmetric interaction promoting microtubule elongation. Nat. Commun. 5, 5072.

Murray, A. W. (1991). Cell cycle extracts. Methods Cell Biol. 36, 581-605.

Newport, J. W. and Kirschner, M. W. (1984). Regulation of the cell cycle during early Xenopus development. Cell 37, 731-742.

O’Brien, L. L., Albee, A. J., Liu, L., Tao, W., Dobrzyn, P., Lizarraga, S. B. and Wiese, C. (2005). The Xenopus TACC homologue, maskin, functions in mitotic spindle assembly. Mol. Biol. Cell 16, 2836-2847.

Ohi, R., Burbank, K., Liu, Q. and Mitchison, T. J. (2007). Nonredundant functions of Kinesin-13s during meiotic spindle assembly. Curr. Biol. 17, 953-959.

Peset, I., Seiler, J., Sardon, T., Bejarano, L. A., Rybina, S. and Vernos, I. (2005). Function and regulation of Maskin, a TACC family protein, in microtubule growth during mitosis. J. Cell Biol. 170, 1057-1066.

Sampath, S. C., Ohi, R., Leismann, O., Salic, A., Pozniakovski, A. and Funabiki, H. (2004). The chromosomal passenger complex is required for chromatininduced microtubule stabilization and spindle assembly. Cell 118, 187-202.

Sardon, T., Peset, I., Petrova, B. and Vernos, I. (2008). Dissecting the role of Aurora A during spindle assembly. EMBO J. 27, 2567-2579.

Tanenbaum, M. E. and Medema, R. H. (2010). Mechanisms of centrosome separation and bipolar spindle assembly. Dev. Cell 19, 797-806.

Toso, A., Winter, J. R., Garrod, A. J., Amaro, A. C., Meraldi, P. and McAinsh, A. D. (2009). Kinetochore-generated pushing forces separate centrosomes during bipolar spindle assembly. J. Cell Biol. 184, 365-372.

Verde, F., Labbe, J.-C., Doree, M. and Karsenti, E. (1990). Regulation of microtubule dynamics by cdc2 protein kinase in cell-free extracts of Xenopus eggs. Nature 343, 233-238.

Wilbur, J. D. and Heald, R. (2013). Mitotic spindle scaling during Xenopus development by kif2a and importin $\alpha$. Elife 2, e00290.

Wittmann, T. and Hyman, T. (1999). Recombinant p50/dynamitin as a tool to examine the role of dynactin in intracellular processes. Methods Cell Biol. 61, 137-143

Wuhr, M., Tan, E. S., Parker, S. K., Detrich, H. W., III and Mitchison, T. J. (2010). A model for cleavage plane determination in early amphibian and fish embryos. Curr. Biol. 20, 2040-2045. 